\title{
High-Throughput Multiplexed Quantitation of Protein Aggregation and Cytotoxicity in a Huntington's Disease Model
}

\author{
Steven A. Titus, Noel Southall, Juan Marugan, Christopher P. Austin and Wei Zheng* \\ National Center for Advancing Translational Sciences, National Institutes of Health. 9800 Medical Center Drive, \\ Bethesda, MD 20892, USA
}

\begin{abstract}
A hallmark of Huntington's disease is the presence of a large polyglutamine expansion in the first exon of the Huntingtin protein and the propensity of protein aggregation by the mutant proteins. Aberrant protein aggregation also occurs in other polyglutamine expansion disorders, as well as in other neurodegenerative diseases including Parkinson's, Alzheimer's, and prion diseases. However, the pathophysiological role of these aggregates in the cell death that characterizes the diseases remains unclear. Identification of small molecule probes that modulate protein aggregation and cytotoxicity caused by aggregated proteins may greatly facilitate the studies on pathogenesis of these diseases and potentially lead to development of new therapies. Based on a detergent insoluble property of the Huntingtin protein aggregates, we have developed a homogenous assay to rapidly quantitate the levels of protein aggregates in a cellular model of Huntington's disease. The protein aggregation assay has also been multiplexed with a protease release assay for the measurement of cytotoxicity resulting from aggregated proteins in the same cells. Through a testing screen of a compound library, we have demonstrated that this multiplexed cytotoxicity and protein aggregation assay has ability to identify active compounds that prevent cell death and/or modulate protein aggregation in cells of the Huntington's disease model. Therefore, this multiplexed screening approach is also useful for development of high-throughput screening assays for other neurodegenerative diseases involving protein aggregation.
\end{abstract}

Keywords: Huntington's disease, protein aggregation, high-throughput screen, polyglutamine expansion, multiplex assay.

\section{INTRODUCTION}

Protein aggregation occurs in several neurodegenerative disorders including Huntington's disease, Alzheimer's disease, Parkinson's disease, Amyotrophic Lateral Sclerosis, and Prion disease. Huntington's disease has been reported to be caused by an abnormally long expansion of a CAG trinucleotide repeat located in exon 1 of the Huntington (HTT) gene although many other factors have been investigated [1]. The expansion of CAG trinucleotide results in a HTT protein bearing a long stretch of polyglutamine residues (PolyQ). The severity and time of onset of the disease are directly proportional to length of the PolyQ expansion [2]. The formation of intranuclear inclusions by the HTT protein with long PolyQ expansion is a characteristic hallmark of the disease. While the question of whether the aggregates in cells are toxic or even protective is still debated [3,4], small molecule modulators that prevent the formation of protein aggregates should be useful research tools for further exploring the pathology of the disease as well as serving as lead compounds for potential drug development.

Existing assays for measuring protein aggregation are limited to lower-throughput or non-quantitative methods

*Address correspondence to this author at the National Center for Advancing Translational Sciences/NIH, 9800 Medical Center Drive, MSC: 3370, Bethesda, MD 20892-3370, USA; Tel: (301)827-6727; Fax: (301)217-5728; E-mail: wzheng@mail.nih.gov
[5-8]. Protein aggregates in cells are commonly detected by a direct staining with a fluorescent dye such as Congo Red [9], immunostaining with an antibody against to the aggregated protein [10], expression of an epitope tag, or a fluorescent protein-tagged fusion protein. Many protein aggregation assays require microscopic or an imaging-based instrument for detection and thus the throughput for compound screening is relatively low. Although imaging instrumentation for high content screening (HCS) has advanced remarkably in the last decade, the limited speed of data acquisition and data processing prevent HCS from being used in protein aggregation assays for screening of very large compound collections $[11,12]$. Here we report a novel homogenous protein aggregation assay using a laser scanning cytometer plate reader to quantitate the protein aggregates formed in the cells by expression of GFP-PolyQ fusion proteins. This assay has been multiplexed with a cytotoxicity assay for sequential measurements of cell viability and protein aggregates in a cell model of Huntington's disease that has been used in a testing screen of a compound library. Our results indicate that this multiplexed assay for protein aggregation and cytotoxicity is a robust and reliable assay method for high throughput screening.

\section{MATERIALS AND METHODS}

\section{Materials}

Tebufenozide and other chemicals were purchased from Sigma-Aldrich (St. Louis, MO). DMEM medium and other 
cell culture supplies were obtained from Invitrogen (Carlsbad, CA). The 1536-well compound plates and black/clear assay plates were purchased from Greiner Bioone (Monroe, NC). The CytotoxGlo protease release assay was purchased from Promega (Madison, WI), and the ATPLite assay was purchased from PerkinElmer (Waltham, MA).

\section{Cell Culture and Plating}

Rat pheochromocytoma PC12 cell lines harboring HTTQ103 or Q25 (a wild type control) fused to GFP under a tebufenozide inducible promoter were kindly provided by Dr. Eric Schweitzer $[12,13]$. Cells were maintained in the phenol red free DMEM medium at $37^{\circ} \mathrm{C}$ under a humidified atmosphere containing $5 \% \mathrm{CO}_{2}$ and $95 \%$ air. The medium contained 5\% calf serum, 5\% horse serum (both from HyClone, Logan UT), $250 \mu \mathrm{g} / \mathrm{ml}$ geneticin, $1 \times$ pen/strep, and 2 $\mathrm{mM}$ L-glutamine. Cells were passaged when they reached 80 to $90 \%$ confluency. The PC-12 cell lines have stringent growth conditions; if the cells become too confluent over time, the entire population will perish, and if the cells are seeded too sparsely, they will not grow efficiently. Phenol red was shown to slightly inhibit the luminescent signals so phenol red free media was chosen for the assay.

Cells were plated at a density of 1000 to 1500 cells/well in black, clear bottom, tissue culture treated and low base, 1536 well plates in $5 \mu \mathrm{l} /$ well of complete DMEM media except geneticin using a Multidrop Combi dispenser (Thermo Scientific, Waltham, MA) and incubated for $24 \mathrm{hr}$ at $37^{\circ} \mathrm{C}$ under a humidified atmosphere containing $5 \% \mathrm{CO}_{2}$. Tebufenozide was used at $200 \mathrm{nM}$ final concentration to induce expression of Q103-GFP and Q25-GFP fusion proteins in these PC12 cell lines. Both tebufenozide and test compounds in DMSO solution were sequentially added to the assay plates at a volume of $23 \mathrm{nl} /$ well using a Kalypsys pintool station. The final concentration of DMSO in assay plates was $0.46 \%$ and no significant cytotoxicity was observed in cells treated with this concentration of DMSO (data not shown). The assay plates were incubated at $37^{\circ} \mathrm{C}$ for 48 hours before being assayed in the following multiplexed dual read assay format.

\section{Measurement of Cytotoxicity Caused by Protein Aggre- gation}

Significant cytotoxicity and subsequent aggregation of the Q103-GFP fusion proteins were found after the induction of the fusion protein expression in the PC12 cell line. Cytotoxicity was first determined by a CytotoxGlo protease release assay kit by quantitating the activity of proteases released from impaired cell membrane. Briefly, 2ul/well of the reagent mixture from the protease assay kit was added to assay plates and the plates were centrifuged at 1500 RPM for $30 \mathrm{sec}$ to remove air bubbles which would affect measurement of the luminescent signal. After 10 minutes of incubation at room temperature, the assay plates were measured in a luminescent mode of Viewlux plate reader for the activity of protease released to the media. The protease activity in the media increases with the increased damaged integrity of plasma membrane due to the cytotoxicity of protein aggregation in the Q103-GFP PC12 cell line.

\section{Measurement of Protein Aggregates}

Cells in the same assay plates after the above cytotoxicity measurement were then treated with $1 \mu \mathrm{l} /$ well of TritonX100 ( $0.25 \%$ final) for cell lysis that dissolved soluble proteins from the bottom of assay plates. Only the aggregated proteins that were insoluble to the detergent treatment still attached to the bottom of assay plates after this detergent treatment. The assay plates were then measured by a bottom reading mode in an Acumen Explorer reader (TTP Labtech Inc., Melbourn, UK), a laser scanning cytometer, for quantitation of the numbers of Q103-GFP aggregates. The measurement parameters in the Acumen Explorer reader included (1) excitation with a $488 \mathrm{~nm}$ laser and emission at 500-530 $\mathrm{nm}$, (2) PMT voltage of 450 volts, and (3) the number of fluorescent objects in size of 1 to $25 \mu \mathrm{m}$ with a signal of 6 standard deviations above background. The number of fluorescent objects was directly proportional to the severity of protein aggregation observed under a fluorescence microscope in the Q103-GFP cell line.

\section{Compound Libraries}

A library collection of 220,581 compounds was screened at four compound concentrations. All compounds were dissolved in DMSO at $10 \mathrm{mM}$ as stock solutions that were serially diluted in DMSO in 384-well plates at a ratio of 1:5. The four sets of 384-well inter-plate dilution plates were subsequently reformatted into one set of 1536-well compound plates at $7 \mu \mathrm{l} /$ well using a Cybi-well dispensing station with a 384-well head (Cybio, Inc.). The final compound concentrations in $5 \mu \mathrm{l} /$ well assay volume ranged from $500 \mathrm{nM}$ to 40 $\mu \mathrm{M}$ in our screen.

\section{Instruments for Liquid Handling and Plate Detection}

The reagent dispensing and compound transfer in 1536well plates were handled by automated work stations. A BioRAPTR work station (Beckman Coulter, Brea, CA) using solenoid valves for dispensing was employed to deliver reagents to 1536 -well plates ranging from 0.5 to $5 \mu \mathrm{l}$ per well. A pintool compound transfer station was used to transfer 23 $\mathrm{nl}$ compounds in DMSO solution to the media of $5 \mu \mathrm{l} /$ well cells in 1536-well assay plates.

\section{Data Analysis}

The primary screening data was first analyzed using customized software developed internally [21]. Clustering analysis of active compounds from the primary screening was performed with Leadscope ${ }^{\circledR}$ Hosted Client (Leadscope Inc., Columbus, OH). The results of concentration response curves were analyzed and plotted with the Prism program (GraphPad Software, San Diego, CA).

\section{RESULTS}

\section{Measurement of Protein Aggregates}

The Q103-GFP cell line has been used in previous screening assays as a cellular model of HD $[12,13]$. We used a PC12 cell line that inducibly expresses the first 17 amino acids of exon 1 of the HTT gene with 103 glutamines fused to a GFP reporter. The induction of Q103-GFP expression in the cells causes the formation of perinuclear, fluorescent aggregates (Fig. 1A). The size and fluorescence inten- 
A

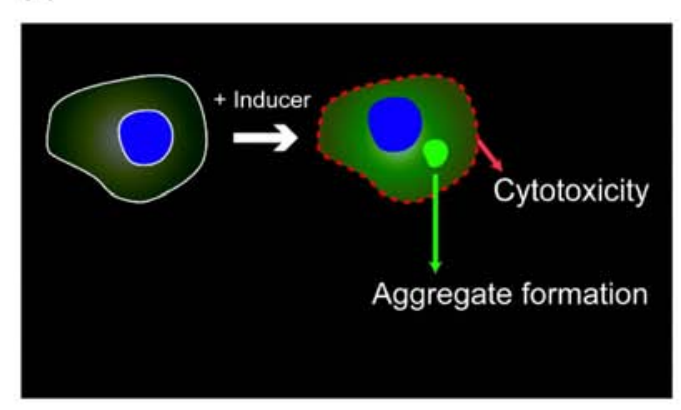

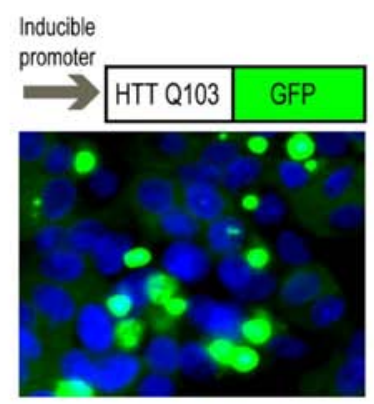

B

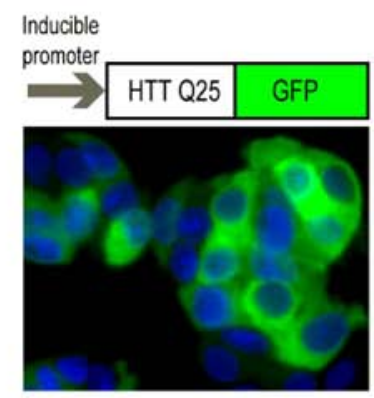

Fig. (1). Schematic representation of a cell based Huntington's disease model. (A) In a Q103-GFP PC12 cell line, induction of Q103-GFP fusion protein expression causes formation of protein aggregates and resultant cytotoxicity. Typical screening assays measure either the cytotoxicity caused by the expression of Q103-GFP fusion proteins or protein aggregates. (B) In a Q25-GFP control cell line, the Q25-GFP fusion proteins are diffusely distributed in cytosol that do not form aggregates and are not toxic to cells. The images of Q103-GFP and Q25GFP in (A) and (B) were obtained with a fluorescence confocal microscope using a 40× objective. Nuclei (blue color) were stained with Hoechst 33342 and the GFP fusion proteins (either soluble proteins or aggregates) are shown in green color.

sity of these aggregates increase with cell incubation time in the presence of inducer. Additionally, expression of the Q103-GFP fusion protein is cytotoxic, resulting in approximately $40-50 \%$ cell death after $48 \mathrm{hr}$ induction of expression of the fusion protein [13]. However, the protein aggregation and resultant cytotoxicity are not observed in the cells of a control line with Q25-GFP fusion protein (Fig. 1B) after 48 $\mathrm{hr}$ incubation with the inducer.

It is challenging to measure these protein aggregates in a high throughput screening format as all existing assays require extensive manipulation of the samples including several complicated assay steps. During the course of assay development, we observed that the fluorescent GFP aggregates in Q103-GFP cells remained on the bottom of assay plates after cell lysis by $0.25 \%$ Triton X-100. In contrast, no fluorescent signal was observed on the bottom of assay plates with the Q25-GFP control cells after the same detergent treatment (Fig. 2). The results indicate that the soluble fusion proteins were released into the solution after the detergent treatment, while the protein aggregates were insoluble to detergent and remained on the bottom of assay plates. Importantly, this finding enabled us to develop a homogenous assay for the rapid quantitation of protein aggregates without any cell wash steps. In addition, we found that our observation is similar to a previous report that the HTT polyQ aggregates were resistant to detergent treatment whereas nonaggregated polyQ proteins were solublized by detergent [14].

We also found that the fluorescence intensities from whole wells of assay plates measured by a conventional plate reader were not able to detect the difference between the Q103-GFP and Q25-GFP control cells. This could be due to the similar expression levels of total GFP fusion proteins in both cell lines and the fluorescence intensities from soluble proteins and aggregates were not distinguishable by measuring a whole well in assay plates. We then used a laser scanning plate cytometer (Acumen Explorer reader) which read from the bottom of assay plates and applied a signal threshold algorithm to identity fluorescent objects (e.g. aggregates) on the bottom of plates against the fluorescent background in solution. We found that the GFP tagged aggregates in Q103GFP cells were distinguishable from the soluble GFP fusion proteins in Q25-GFP control cells after the detergent treatment. Thus, from optimizing the measurement parameters, only the intensity of fluorescent objects ranging in size of 1 to $25 \mu \mathrm{m}$ were counted from the bottom of assay plates. The $\mathrm{EC}_{50}$ values of tebufenozide, an inducer for the fusion protein expression, were 25.5 and $28.9 \mathrm{nM}$ in the Q103-GFP cells prior to and after the detergent treatment, respectively (Fig. $\mathbf{2 A}$ ). The result indicated that the detergent treatment did not change the fluorescence signals from Q103-GFP fusion proteins. Although the concentration response of tebufenozide in Q25-GFP cells was observed before the detergent treatment, no detectable signal was found by using the Acumen Explorer reader because the Q25-GFP fusion proteins were dispersed in solution after the detergent treatment (Fig. 2B). Thus, this protein aggregation assay based on the laser scanning cytometer detection is able to distinguish the aggregated fusion proteins from the un-aggregated proteins in assay plates after the treatment with $0.25 \%$ Triton X-100 detergent.

\section{Measurement of Cytotoxicity with a Protease Release Assay}

Expression of long polyglutamine expansions such as in the Q103-GFP cells has been shown to be a cause of protein aggregation and resulting cell death in many cell types [15]. Compounds that inhibit the cytotoxicity of polyglutamine expression are of interest as research tools to study the pathophysiology of many diseases related to protein aggregation. In order to identify lead compounds which inhibit cytotoxicity caused by expression of long polyglutamine expansions, we multiplexed the assay readout to measure both protein aggregation and cytotoxicity in the same well of the same assay plate.

In Q103-GFP cells, only a subpopulation of cells (usually $\sim 40-50 \%$ ) is adversely affected after $48 \mathrm{hr}$ induction of expression of Q103-GFP proteins. Previous reports indicated that signal-to-basal ratios of MTT or LDH release assays in these cells were not robust with lower signal-to-basal ratios [13]. We initially tried an ATP content assay for the measurement of ATP levels in viable cells with a luminescence readout. The signal-to-basal ratio was around 1.5 to 2 fold, presumably due to the large percentage of viable cells (Fig. 3A). 


\section{Q103-GFP cells}
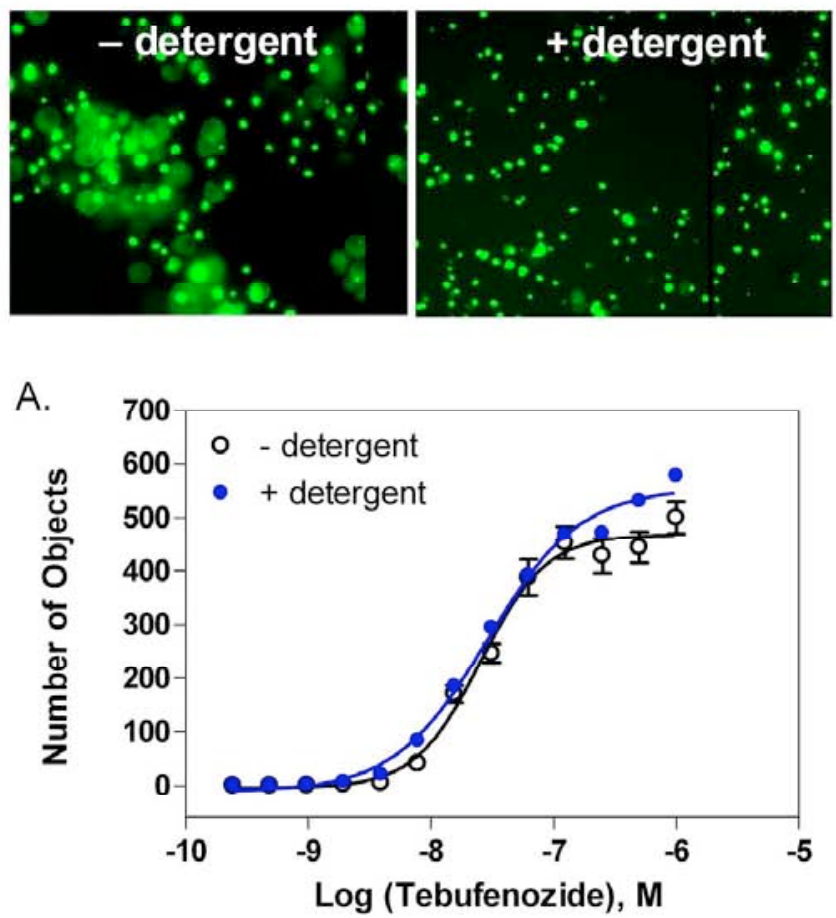

Q25-GFP cells

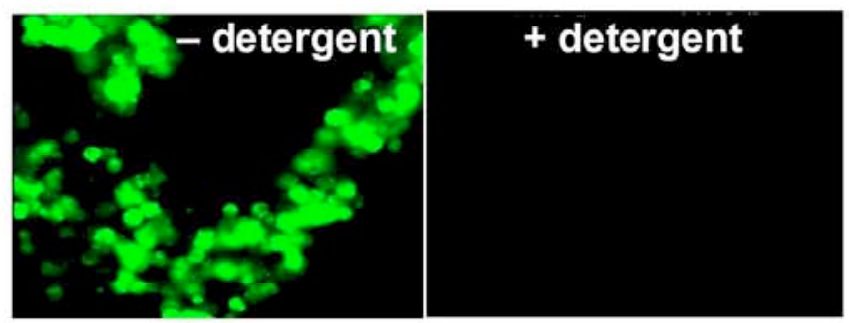

B.

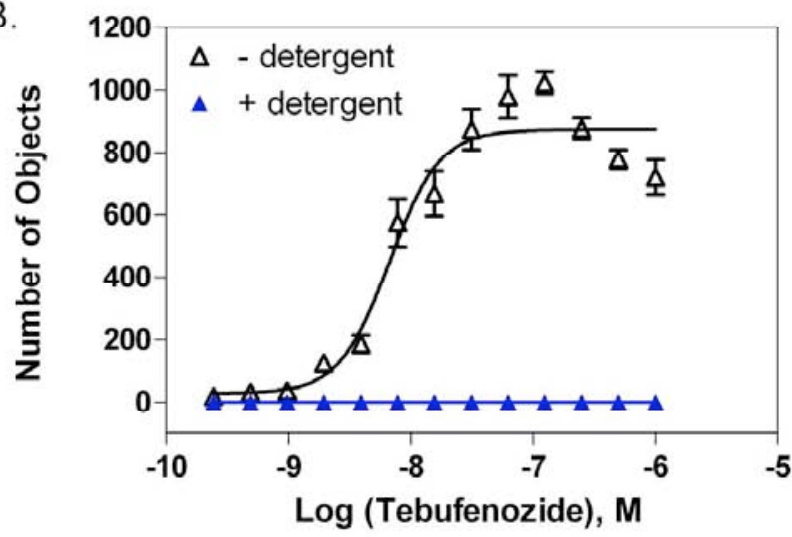

Fig. (2). Differentiation of the fluorescent signals from aggregated Q103-GFP and non-aggregated G25-GFP fusion proteins by a laser scanning cytometer plate reader before and after detergent treatment. Microscopic images were captured using a $20 \times$ objective on an inverted epifluorescence microscope. In Q103-GFP cells, the detergent treatment did not disrupt the protein aggregates attached to the bottom of an assay plate, while soluble proteins in Q25-GFP control cells diffused away from the bottom of the plate and were not visible after detergent treatment. (A) In Q103-GFP cells, the concentration response curves of the inducer tebufenozide were similar in the presence and absence of detergent. (B) In Q25-GFP control cells, no fluorescent signal was detected after detergent treatment because the fusion protein was dispersed into the medium and only the fluorescence signal at the bottom of an assay plate was measured by the reader.

We then applied a protease release assay that measures the activity of released proteases from damaged plasma membrane due the toxicity of Q103-GFP proteins. We found that the signal-to-basal ratio of this protease release assay in Q103-GFP cells was 11.1 fold, indicative of a robust assay for high throughput screening (Fig. 3A). The high signal-tobasal ratio was found in this protease release assay that may be due to the lower basal signal in this assay compared with the high basal signal in the ATP content assay [16]. The cellular proteases only leak out to media when the integrity of plasma membrane is perturbed in apoptotic or necrotic cells. The cytotoxicity of GFP-Q103 protein expression after $48 \mathrm{hr}$ induction with the inducer tebufenozide was much more significant compared with that of $24 \mathrm{hr}$ induction, which correlated with the time course of protein aggregation formation detected by the above assay (Fig. 3B).

\section{Multiplexing Cytotoxicity Measurement with Detection of Protein Aggregation}

Because the measurements of cytotoxicity and protein aggregation described in the above experiments utilize two different detection modes (e.g. luminescence and fluorescence intensity, respectively), we tried to multiplex them into sequential measurements in a single assay plate. The main advantage for using the same assay plate to measure compound effects on both cytotoxicity and protein aggregation is that the compound activities in these two assays are determined from the cells treated under identical experimental conditions. The results from such a multiplexed assay would be more comparable for the similarity or difference in compound activities on protein aggregation and cytotoxicity caused by the aggregated proteins. This multiplexed assay also has very few steps and is therefore relatively simple for the robotic screening (Table 1). Briefly, the cells are seeded into a 1536 well plate and incubated for one day. Expression of the Q103-GFP or Q25-GFP fusion proteins is induced by the addition of $200 \mathrm{nM}$ tebufenozide (with or without library compound addition). After a $48 \mathrm{hr}$ treatment with the inducer, cytotoxicity is first measured by the protease release assay in the luminescence detection mode with a single reagent addition step. After the cytotoxicity measurement, the assay plates are treated with detergent to lyse the cells that subsequently allows all soluble proteins to diffuse into the surrounding media. The fluorescent protein aggregates are then measured using a laser scanning plate cytometer (Fig. 4).

\section{Assay Validation and Compound Library Screen}

Using a DMSO plate as a control, we found that the signal-to-basal ratios for cytotoxicity and protein aggregate measurements were 9.6 and 250 fold, the CVs were $7.5 \%$ and $9.8 \%$, and the $Z^{\prime}$ factors were 0.80 and 0.83 , respectively (Figs. 3C and 3D). The results from this multiplexed 


\section{A. Cytotoxicity assays}

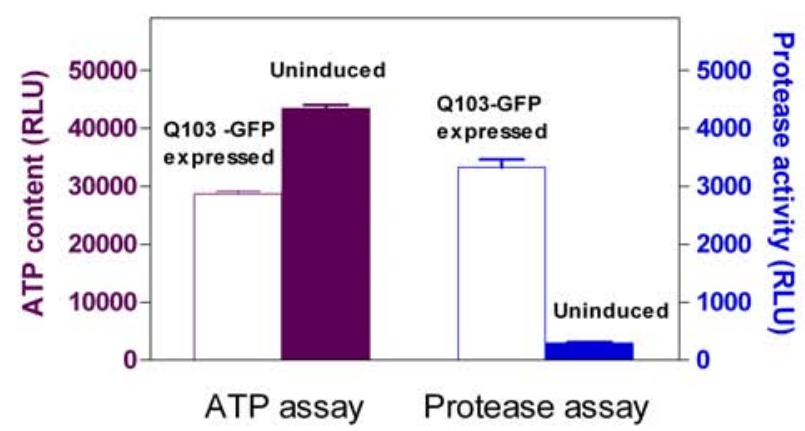

C. Protease release assay

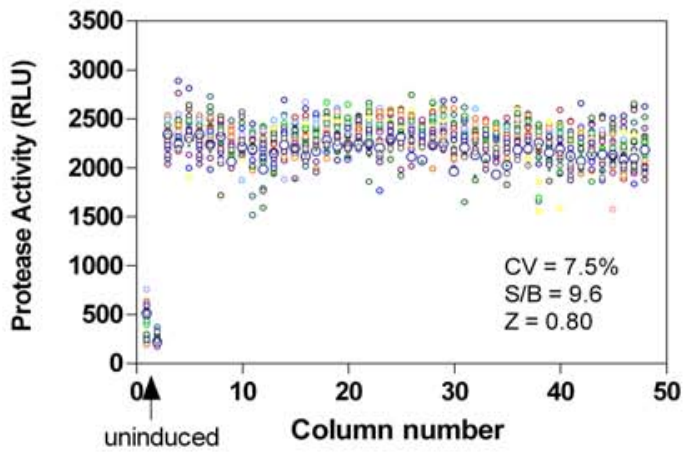

B. $24 \mathrm{hr}$ vs. $48 \mathrm{hr}$ Induction

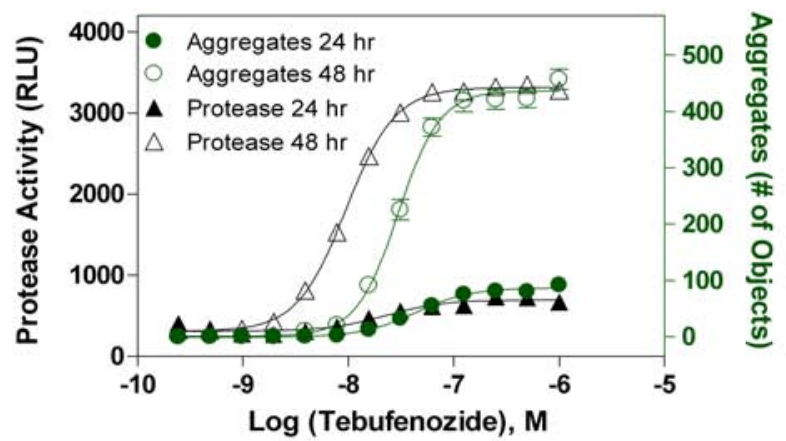

D. Aggregation assay

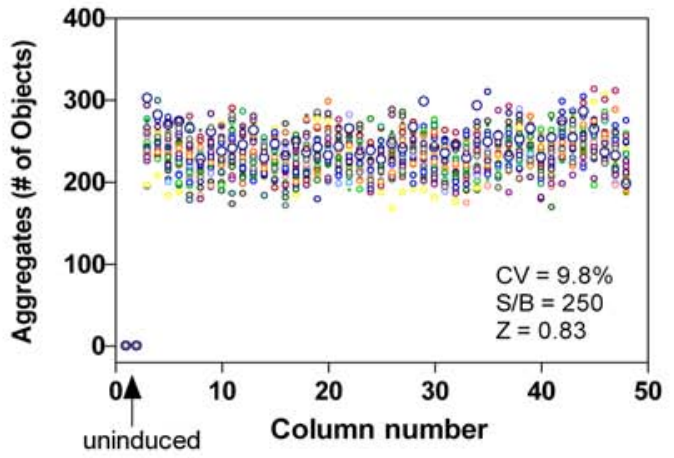

Fig. (3). (A) Comparison of an ATP content assay and a protease release assay for measurements of cytotoxicity resulted from the expression of Q103-GFP fusion proteins in the Q103 PC12 cells. The expression of Q103-GFP fusion proteins in these cells was induced by 48 hr treatment with the inducer tebufenozide. (A) In the ATP content assay, 30 to $50 \%$ reduction in ATP levels caused by the fusion protein cytotoxicity was observed in the cells treated with the inducer tebufenozide compared with that in untreated cells, resulting in a signal-to-basal (S/B) ratio of 1.4 fold. In the protease release assay, the protease activity due to the cytotoxicity was dramatically increased in the cells treated with the inducer tebufenozide compared with that in untreated cells $(n=4$ for each data point). The average S/B ratio was 9 fold. Thus, the protease assay produced a better assay window and was selected for the measurement of cytotoxicity in our experiments. (B) Concentration response curves of inducer tebufenozide determined in Q103-GFP cells after 24 or $48 \mathrm{hr}$ treatment. The average S/B ratios in both protease and aggregation assays were much higher after $48 \mathrm{hr}$ incubation with the inducer tebufenozide than these after $24 \mathrm{hr}$ incubation. The EC $\mathrm{E}_{50}$ values of tebufenozide determined from the protease release cytotoxicity assay and protein aggregation assay were 9.5 and $29.6 \mathrm{nM}$, respectively ( $\mathrm{n}=12$ for each data point). (C) Scatter plot of a DMSO plate test in the protease release assay. (D) Scatter plot of a DMSO plate test in protein aggregation assay. Both results in C and $\mathrm{D}$ were obtained from the same assay plate in a multiplexed assay experiment. In both panel C and D, the wells in columns 1 and 2 were not treated with inducer (e.g. negative control) and wells in columns 3 through 48 were treated with $200 \mathrm{nM}$ Tebufenozide for 48 hours. Uninduced cells exhibited virtually no detergent insensitive fluorescence signal (D). All the wells also received $0.46 \%$ DMSO, a solvent for compounds.

Table 1. Protocol for Multiplexed Protein Aggregation and Cytotoxicity Assay in 1536-Well Plate Format

\begin{tabular}{|c|c|c|c|}
\hline Step & Parameter & Value & Description \\
\hline \hline 1 & Cell plating & $5 \mu \mathrm{l} / \mathrm{well}$ & at $37^{\circ} \mathrm{C}$ with $5 \% \mathrm{CO}_{2}$ \\
\hline 2 & Incubation & $24 \mathrm{hr}$ & at $37^{\circ} \mathrm{C}$ and $5 \% \mathrm{CO}_{2}$ \\
\hline 3 & Compound addition & $0.023 \mu 1 /$ well & Compounds in DMSO solution and $200 \mathrm{nM}$ Tebufenozide (inducer) \\
\hline 4 & Incubation & $48 \mathrm{hr}$ & reagent mixture from the protease assay kit \\
\hline 5 & Reagent-1 & $2 \mu 1 / \mathrm{well}$ & at ${ }^{\circ} \mathrm{C}$ \\
\hline 6 & Incubation & $10 \mathrm{~min}$ & Viewlux plate reader \\
\hline 7 & Plate reading-1 & luminescence & $0.25 \%$ Triton $\mathrm{X}-100$ (final) \\
\hline 8 & Reagent-2 (cell lysis) & $2 \mu 1 / \mathrm{well}$ & room temperature \\
\hline 9 & Incubation & $30 \mathrm{~min}$ & Acumen Explore reader to count \# of aggregates \\
\hline 10 & Plate reading-2 & Ex=488 nm, Em=500-530nm & \\
\hline
\end{tabular}




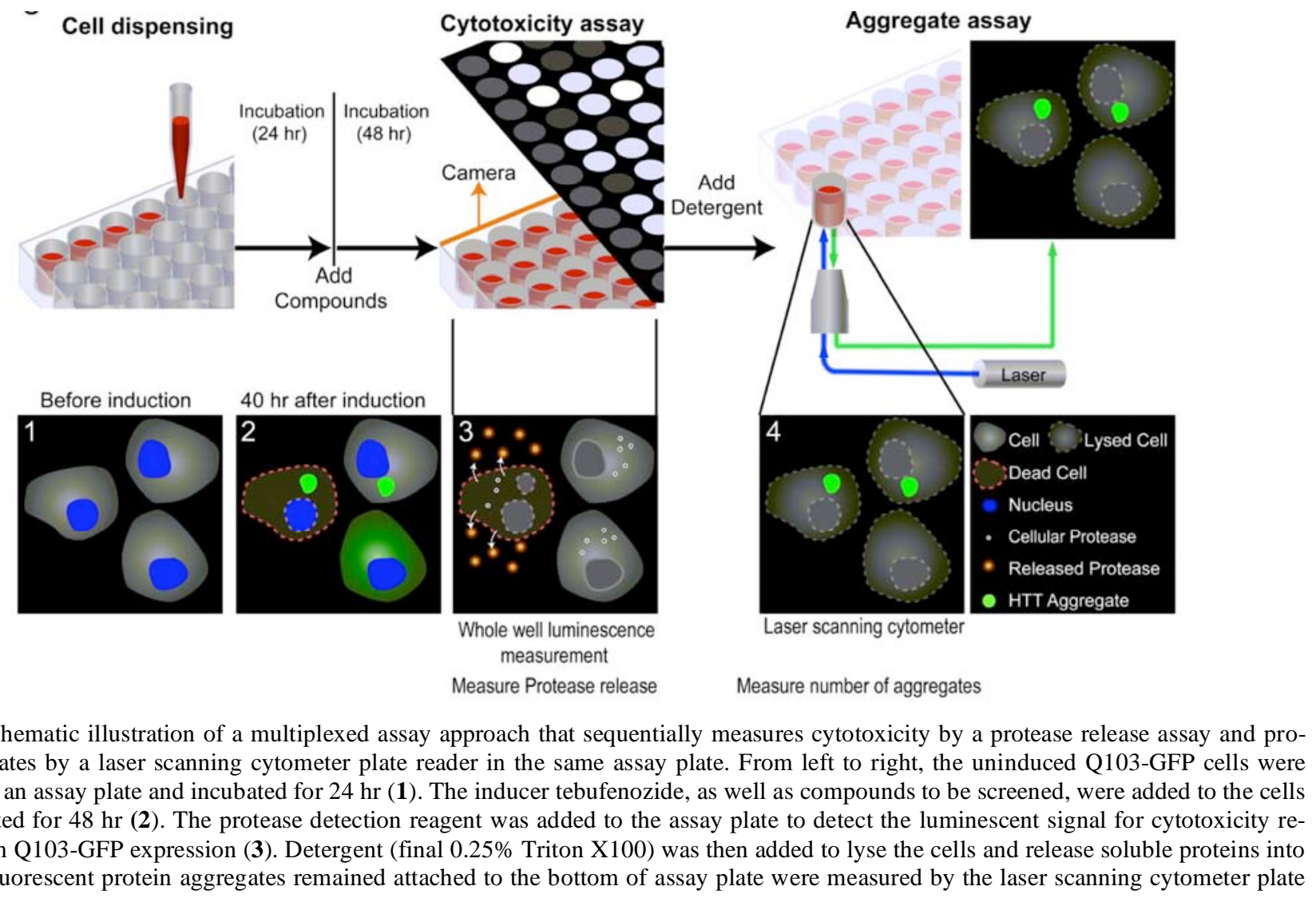

Fig. (4). Schematic illustration of a multiplexed assay approach that sequentially measures cytotoxicity by a protease release assay and protein aggregates by a laser scanning cytometer plate reader in the same assay plate. From left to right, the uninduced Q103-GFP cells were seeded into an assay plate and incubated for $24 \mathrm{hr}(\mathbf{1})$. The inducer tebufenozide, as well as compounds to be screened, were added to the cells and incubated for $48 \mathrm{hr}(\mathbf{2})$. The protease detection reagent was added to the assay plate to detect the luminescent signal for cytotoxicity resulting from Q103-GFP expression (3). Detergent (final 0.25\% Triton X100) was then added to lyse the cells and release soluble proteins into solution. Fluorescent protein aggregates remained attached to the bottom of assay plate were measured by the laser scanning cytometer plate reader (4)

assay demonstrate the robustness of assay performance, which is suitable for high throughput screening of large compound collections. We then screened a library of 220,581 small molecule compounds at four concentrations ranging from 0.5 to $40 \mu \mathrm{M}$. Several groups of active compounds with different effects on cytotoxicity and protein aggregation were identified from this screen. The primary screening results have been deposited in PubChem (AID 1471, 1482 and 1688).

Representative compounds identified from the compound library screening are shown in Fig. (5). The cytoprotective compounds identified were classified into three groups; (1) compounds inhibiting cell death but increasing protein aggregation (top panel), (2) compounds inhibiting both cell death and protein aggregation (middle panel), and (3) compounds inhibiting cell death without an effect on protein aggregation (bottom panel). While all three representative compounds exhibited cytoprotective effect in the protease release assay as well as an ATP quantitation assay (data not shown), compound 1 (securinine) increased the number of protein aggregates (green color in the image), compound 2 (hycanthone) decreased the number of protein aggregates and compound 3 (ipriflavone) had no effect on the protein aggregates compared with the control cells. These active compounds could be useful research tools for the further studies on the mechanism of protein aggregation and its relation with cytotoxicity as well as further studies in animal models. Some of these compounds are currently under investigation to identify their potential mechanisms of action. The results of structure-activity studies as well as the potential mechanism of action will be reported in a separate article.

\section{DISCUSSION}

Our goal was to develop an HTS assay that has an ability to interrogate compound effects on both the poly-Q aggregation and resulting cytotoxicity in a cell model of HD. The protein aggregation assay described here has several advantages over previous assays using a standard or confocal fluorescence microscope. First, the high energy excitation used in the laser scanning cytometer significantly improves the signal-to-basal ratio of the measurement. Second, the read time of $\sim 10$ minutes per plate is significantly shorter compared to an automated imaging system which typically requires 45 or more minutes to measure a 1536 well plate. Third, the data processing is conducted in parallel with the image acquisition so no extra time was required for data processing. Fourth, the size of data file for an assay plate, typically $500 \mathrm{~kb}$, is much smaller compared to the image file generated by a microscopic reader. In contrast, the imagingbased HCS screens capture multiple image fields per well which in turn produces large data files; typically 4 to 10 gigabytes per 1536 well plate. Therefore, this protein aggregation assay is convenient and user-friendly for compound screening of large libraries.

The phenotype of toxic gain of function associated with polyglutamine expansions is similarly present in all CAG expansion-related diseases [17]. The formation of protein aggregates observed in these diseases is a slow process whereby the protein filaments slowly undergo conformational changes; presumably evolving from compact $\beta$-sheets, to protofibrils, fibrils, and then aggregate foci, and finally visible aggregates [18]. These stages appear to have differen- 


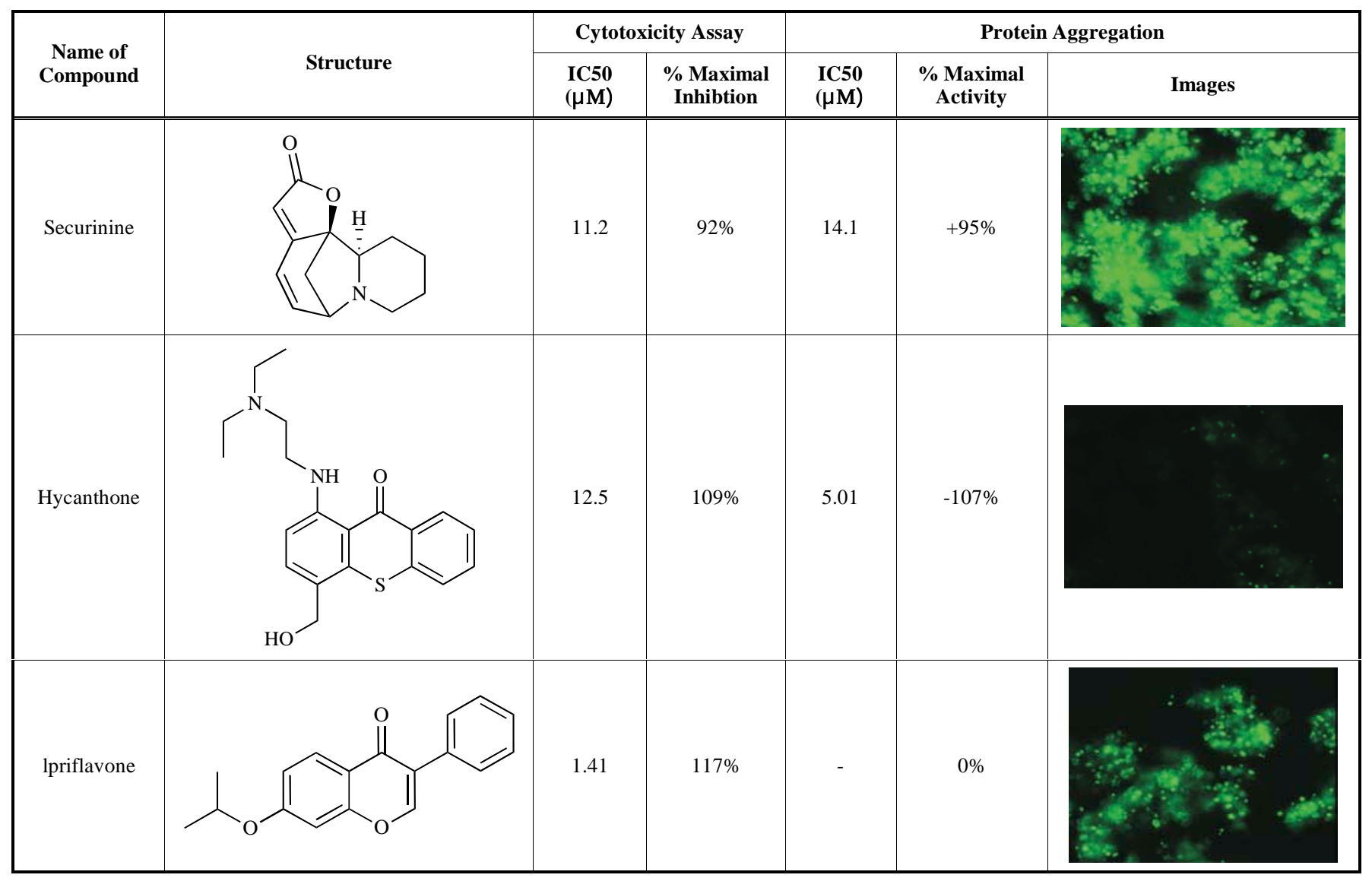

Fig. (5). Three representative compounds that modulate cytotoxicity and protein aggregation identified from the compound library screen using the multiplexed cytotoxicity and protein aggregation assay. All three compounds inhibited the cytotoxicity caused by the expression of Q103-GFP fusion proteins in the Q103-GFP cells. The Compound -1 (on the top panel) had an $\mathrm{IC}_{50}$ of $11.2 \mu \mathrm{M}$ on inhibition of cytotoxicity but it increased the aggregates formation with a potency of $14.1 \mu \mathrm{M}$. Compound -2 (in the middle panel) had an $\mathrm{IC}_{50}$ of $12.5 \mu \mathrm{M}$ on inhibition of cytotoxicity and an $\mathrm{IC}_{50}$ of $5.01 \mu \mathrm{M}$ against the aggregates formation. Compound -3 (on the bottom panel) had an IC $\mathrm{C}_{50}$ of $1.41 \mu \mathrm{M}$ on inhibition of cytotoxicity without effect on protein aggregation. The potency and percentage of maximal control response for cytoprotection and aggregates formation are listed in columns 3 though 6 , respectively. The last column shows the images (magnified by $20 \times$ ) of unlysed cell and their associated GFP aggregates in the presence of $25 \mu \mathrm{M}$ compounds.

tial affects on cytotoxicity [19]. However, it is still unknown at which stage these proteins become detergent insensitive, and how that relates to cytotoxicity. Future studies exploring various parameters such as different time points, detergent concentrations, or different types of detergents may help to better understand of the process and pathogenesis of aggregation formation.

Although 220,581 compounds were screened using this multiplex assay and several compounds identified, an extended library screen with a larger or more diverse compound collection might be needed to identify better lead compounds. Our results from this compound screen validated this multiplex screen assay as a useful method for identifying lead compounds that inhibit protein aggregation and/or reduce cytotoxicity caused by protein aggregates. In addition, this method might also be useful for studying the kinetic features of protein aggregate formation and effect of other cellular proteins on aggregates formations because of the robustness of assay performance and simple assay procedure.

In summary, we have demonstrated a multiplexed assay method which effectively measures cytotoxicity and protein aggregation in a cell-based model of Huntington's disease.
This assay method has been miniaturized into 1536-well plate format to screen large size compound collections for lead identification. This assay method also has the potential utility in screening other cell-based models of neurodegenerative diseases in which protein aggregation or plaque formation is implicated in the disease pathology $[19,20]$.

\section{CONFLICT OF INTEREST}

The authors confirm that this article content has no conflicts of interest.

\section{ACKNOWLEDGEMENTS}

This research was supported by the Molecular Libraries Initiative of the NIH Roadmap for Medical Research (5U54MH084681-02 and MH084839-01) and the Intramural Research Program of National Center for Advancing Translational Sciences, National Institutes of Health. We would like to thank E. Schweitzer for the PC12 cell lines and protocols, D. Leja for artwork, M. DeBernardi for confocal images, and W. Bowen for technical assistance with the Acumen Explorer protocol and manuscript. 


\section{ABBREVIATIONS}

$\begin{array}{ll}\text { HTT } & =\text { Huntingtin gene } \\ \text { HD } & =\text { Huntington's Disease } \\ \text { HTS } & =\text { high throughput screening } \\ \text { LOPAC } & =\begin{array}{l}\text { Library of Pharmacologically Active Com- } \\ \text { pounds }\end{array}\end{array}$

MLPCN $=$ Molecular Libraries Probe Centers Network

\section{REFERENCES}

[1] Varma H. Drug screening for Huntington's disease and other neurodegenerative disorders. Curr Mol Pharmacol 2010; 3(3): 16473.

[2] Walker FO. Huntington's disease. Lancet 2007; 369(9557): 218-28.

[3] Arrasate M, Mitra S, Schweitzer ES, Segal MR, Finkbeiner S. Inclusion body formation reduces levels of mutant huntingtin and the risk of neuronal death. Nature 2004; 431(7010): 805-10.

[4] Imarisio S, Carmichael J, Korolchuk V, et al. Huntington's disease: from pathology and genetics to potential therapies. Biochem $\mathbf{J}$ 2008; 412(2): 191-209.

[5] Apostol BL, Kazantsev A, Raffioni S, et al. A cell-based assay for aggregation inhibitors as therapeutics of polyglutamine-repeat disease and validation in Drosophila. Proc Natl Acad Sci USA 2003; 100(10): 5950-5.

[6] Bodner RA, Outeiro TF, Altmann S, et al. From the Cover: Pharmacological promotion of inclusion formation: A therapeutic approach for Huntington's and Parkinson's diseases. Proc Natl Acad Sci USA 2006; 103(11): 4246-51.

[7] Heiser V, Engemann S, Brocker W, et al. Identification of benzothiazoles as potential polyglutamine aggregation inhibitors of Huntington's disease by using an automated filter retardation assay. Proc Natl Acad Sci USA 2002; 99(90004): 16400-6.
[8] Pollitt SK, Pallos J, Shao J, et al. A rapid cellular FRET assay of polyglutamine aggregation identifies a novel inhibitor. Neuron 2003; 40(4): 685-94

[9] Scherzinger E, Lurz R, Turmaine M, et al. Huntingtin-encoded polyglutamine expansions form amyloid-like protein aggregates in vitro and in vivo. Cell 1997; 90(3): 549-58.

[10] Gutekunst CA, Li SH, Yi H, et al. Nuclear and neuropil aggregates in Huntington's disease: Relationship to neuropathology. J Neurosci 1999; 19(7): 2522-34.

[11] Scotter EL, Narayan P, Glass M, Dragunow M. High throughput quantification of mutant huntingtin aggregates. J Neurosci Methods 2008; 171(1): 174-9.

[12] Varma H, Lo DC, Stockwell BR. High throughput screening for neurodegeneration and complex disease phenotypes. Comb Chem High Throughput Screen 2008; 11(3): 238-48

[13] Aiken CT, Tobin AJ, Schweitzer ES. A cell-based screen for drugs to treat Huntington's disease. Neurobiol Dis 2004; 16(3): 546-55.

[14] Kazantsev A, Preisinger E, Dranovsky A, Goldgaber D, Housman D. Insoluble detergent-resistant aggregates form between pathological and nonpathological lengths of polyglutamine in mammalian cells. Proc Natl Acad Sci USA 1999; 96(20): 11404-9.

[15] Li SH, Li XJ. Huntingtin and its role in neuronal degeneration. Neuroscientist 2004; 10(5): 467-75.

[16] Cho MH, Niles A, Huang R, et al. A bioluminescent cytotoxicity assay for assessment of membrane integrity using a proteolytic biomarker. Toxicol in Vitro 2008; 22(4): 1099-106.

[17] Orr HT, Zoghbi HY. Trinucleotide repeat disorders. Ann Rev Neurosci 2007; 30: 575-621.

[18] Bates G. Huntingtin aggregation and toxicity in Huntington's disease. Lancet 2003; 361(9369): 1642-4.

[19] Bucciantini M, Giannoni E, Chiti F, et al. Inherent toxicity of aggregates implies a common mechanism for protein misfolding diseases. Nature 2002; 416(6880): 507-11.

[20] Stefani M, Dobson CM. Protein aggregation and aggregate toxicity: New insights into protein folding, misfolding diseases and biological evolution. J Mol Med 2003; 81(11): 678-99.

[21] Wang Y, Jadhav A, Southal N, Huang R, Nguyen DT. A grid algorithm for high throughput fitting of dose-response curve data. Curr Chem Genom 2010; 4: 57-66.

(C) Titus et al.; Licensee Bentham Open.

This is an open access article licensed under the terms of the Creative Commons Attribution Non-Commercial License (http://creativecommons.org/licenses/by-nc/3.0/) which permits unrestricted, non-commercial use, distribution and reproduction in any medium, provided the work is properly cited. 